\title{
Conflictos éticos en autoría de las publicaciones científicas del área médica
}

\author{
Karla Moënne $B^{1}$, Cristian García B. ${ }^{1^{\star}}$
}

1. Comité de Ética, Sociedad Chilena de Radiología. Santiago, Chile.

La enseñanza de la ética es un componente clave para contar con profesionales médicos que enfrenten adecuadamente los desafíos y eventuales conflictos éticos en el ejercicio de la profesión, tanto en la relación con los pacientes como en todas las otras áreas del desarrollo profesional.

En este manuscrito abordaremos el tema de la autoría responsable y los desafíos éticos en las publicaciones científicas del área médica, incluidas las radiológicas, como una labor de difusión del conocimiento que debiera ser parte del desarrollo profesional de todos los médicos.

En el proceso de comunicación del conocimiento científico, la autoría tiene cada vez más trascendencia, ya que confiere crédito a lo que hacemos y puede tener importantes beneficios académicos, sociales y hasta financieros para los autores. En relación con esto, se ha visto un importante aumento en el número de autores de los trabajos o publicaciones científicas, lo que se ha convertido en un tema cada vez más complejo. Esto se denomina autoría múltiple, aunque aún no se ha consensuado su definición. Si bien este aumento en el número de autores pudiera explicarse en gran medida por el progreso científico, asociado al aumento de la investigación en equipo y multidisciplinaria, a veces el número de autores es desproporcionado en relación con el contenido del trabajo y el tipo de investigación efectuada.

Queda sin resolver la cuestión central, que es la naturaleza y cantidad de contribuciones que son necesarias para calificar o alcanzar la condición de autor de un artículo científico.

El inicio de cada una de las investigaciones y sus respectivas publicaciones se remonta al momento en que alguna $o$ algunas personas asumen que tienen algo que comunicar a la comunidad científica; la publicación de un trabajo científico habitualmente finaliza después de un largo proceso e incluye una responsabilidad ética para cada uno de los investiga- dores, en los diversos roles de cada uno de ellos. El correcto uso de las autorías establece responsabilidad y credibilidad respecto de la información científica comunicada en las publicaciones.

Existen organismos internacionales que regulan los aspectos éticos de las publicaciones científicas, destacando el "Comité Internacional de Editores de Revistas Médicas" (ICMJE), que constituye un referente en el tema. Recomienda que la autoría se base en los siguientes criterios:

1. Aportar contribuciones sustanciales a la concepción o diseño del estudio y a la adquisición, análisis o interpretación de los datos para el trabajo.

2. Participar en la redacción del trabajo o en su revisión crítica, contribuyendo a su contenido intelectual.

3. Aprobar la versión final que será enviada para publicación

4. Asumir la responsabilidad pública de todos los aspectos del estudio, asegurando que contestará de manera adecuada cualquier pregunta relacionada con el estudio.

Todos los individuos que cumplan el primer criterio deben tener la oportunidad de participar en la revisión, redacción y aprobación final del manuscrito; la autoría implica no solo la contribución individual a un proyecto de investigación, sino también la responsabilidad conjunta en todo el proyecto. Inversamente, la omisión de un autor que contribuyó significativamente a la investigación supone una apropiación indebida de la autoría intelectual. Como se comentó previamente, el aumento en el número de autores no se acompaña necesariamente de una mejoría en la calidad del contenido de los artículos y, por otra parte, la autoría múltiple no constituye por sí misma una falta a la ética, cuando puede explicarse por razones válidas. Lo que está en duda no es el 
número de autores legítimos, sino la legitimidad de la autoría de cada uno de ellos.

En Chile, la ley $\mathrm{N}^{\circ} 17.336$ regula la propiedad intelectual y protege sus derechos, sin embargo, la autoría científica no obedece a las "leyes del copyright" o "derecho de copia" sino a un aspecto más bien simbólico, como es el reconocimiento de pares.

Debido a que la autoría no especifica qué contribuciones calificaron a un individuo como uno de los autores de un trabajo, la mayor parte de las revistas científicas actualmente solicita y publica información sobre las contribuciones de cada persona nombrada como participante en un trabajo presentado para publicación, al menos en investigación original. Esta exigencia elimina en parte la ambigüedad en cuanto a las contribuciones, pero puede dejar sin resolver con certeza el tema de la cantidad y calidad de la contribución de cada uno de los autores.

Estos criterios de autoría reservan la categoría de autor sólo a los que lo merecen y que por otra parte son quienes asumen la responsabilidad pública del trabajo científico. La determinación de quién califica para autoría le corresponde al grupo de autores y el autor principal será quien debe identificar qué coautores participarán en las distintas secciones del manuscrito y confiar en la integridad de sus contribuciones. Respecto del orden de los autores en la publicación, es conveniente que esto se decida previo al desarrollo del estudio. El autor más relevante es el que aparece en primer lugar y ha realizado la mayor contribución al trabajo. El autor intelectual generalmente va en el último lugar y puede tener igual relevancia que el primero. Ambos pueden actuar como autor correspondiente, encargados de mantener una comunicación directa con el Comité Editorial de la Revista a la que fue enviado el trabajo, resolver las correcciones, etc.

Las discusiones sobre la autoría deberían ocurrir también en el curso del estudio, ya que las contribuciones al trabajo cambian y aquellos que inicialmente se pensó que serían autores, en última instancia pueden no hacer una contribución significativa y, del mismo modo, aquellos que no habían sido considerados inicialmente pueden ser los que aporten con contribuciones importantes. Hay que tener presente que no solo puede cambiar la lista de autores, sino que a menudo el orden de los autores en la lista también puede cambiar a medida que se desarrolla el proyecto.

El concepto de autoría injustificada constituye una falta ética y se aplica cuando el crédito de autor se concede inmerecidamente. Esto puede ocurrir, por ejemplo, cuando algunos grupos de profesionales llegan al acuerdo que, si alguno de ellos escribe un artículo, automáticamente debe agregar el nombre de los demás como autores, aunque estos no hayan intervenido en el trabajo, lo que puede generar un gran aumento en el número de publicaciones para cada uno de ellos.
Dentro de estas malas prácticas, también pueden ocurrir casos de "autorías honoríficas" que otorgan posición de coautor a personas que no han cumplido con los criterios de ICMJE, entre ellos por ejemplo incluir automáticamente al jefe del Departamento.

El concepto de autoría responsable exige además que cada uno de los autores de un manuscrito deba declarar si tiene o no conflicto de intereses en relación con el tema de la investigación. Si los tiene, es imperativo que los conflictos se identifiquen y se explique cuál fue su relación con el trabajo; esto permitirá a los editores, revisores externos y lectores, conocer la influencia que estos autores puedan haber ejercido en la presentación de los resultados y en su interpretación, mejorando la transparencia de los documentos. Desgraciadamente, esto no garantiza que el trabajo esté libre de la influencia de conflictos de interés que no han sido declarados por los autores ni identificados por revisores o editores. En relación con lo anterior, se describe la existencia de "autores fantasmas", que se refiere a individuos que hacen importante contribución al manuscrito, pero no figuran como autores, escondiendo así serios conflictos de intereses. Se estima que en publicaciones biomédicas existiría alrededor de $10 \%$ de este tipo de autores.

Otra figura relacionada con lo antes mencionado es la de "autores invitados", que corresponden a aquellos autores que, sin contribuir intelectualmente con el material de la publicación, aparecen avalando revisiones a favor de un determinado producto. Un método eficaz para evitar o disuadir las autorías injustificadas, es agregar un apartado de agradecimientos, donde se incluya una lista de las personas que contribuyeron en el estudio, pero no califican para ser autores.

Cuando surge una duda o se identifica una transgresión a la ética de las publicaciones científicas, esta debe ser aclarada y solucionada antes que el manuscrito se publique o en su defecto después de que esto ocurra, si la información pertinente se conoce tardíamente. Si se descubre un error $\mathrm{u}$ omisión en la observancia de las normas éticas de las publicaciones científicas, el hecho será considerado como falta grave y los editores deberán informar el hecho en un sitio destacado del número futuro de la revista, además de aplicar eventuales medidas que sean requeridas.

En la publicación del Dr. Humberto Reyes en la Revista Médica de Chile (2007), se destaca que estos temas deben ser tratados con estrictez y respetando las normas éticas de las publicaciones en revistas médicas, porque el mundo científico exige que lo que se publique sea veraz y éticamente correcto, debido a que uno de los propósitos de las revistas médicas es influir en el pensamiento y el actuar clínico de sus lectores, respetando las normas éticas para que así las publicaciones aporten beneficios reales. 


\section{Lectura recomendada}

1. Von Oetinger A, Sadarangani K, Salas S. Conflictos éticos en las autorías de trabajos científicos. Rev Med Chile 2016; 144: 1473-1478.

2. Reyes H, Palma J, Andresen M. Ética de las publicaciones en revistas médicas. Rev Med Chile 2007; 135: 529-533. International Committee of Medical Journal Editors (ICMJE).

http://www.icmje.org/. Consultado 18 septiembre 2019.

3. Isaacs D, Elliot C, Kilham H, Preisz A. The ethics of publishing in medical journals. Paediatric Respiratory
Reviews 2019, en prensa, disponible online: doi: https:// doi.org/10.1016/j.prrv.2019.04.005.

4. Ruíz-Pérez R, Marcos-Cartagena D, Delgado LópezCózar E. La autoría científica en las áreas de ciencia y tecnología. Políticas internacionales y prácticas editoriales en las revistas científicas españolas. Revista Española de Documentación Científica 2014: 37(2): e049. doi: http://dx.doi.org/10.3989/redc.2014.2.1113

5. Gálvez M. Escándalos y prácticas no transparentes en Chile: ¿Algo de qué preocuparnos en Radiología? Rev Chil Radiol. 2017; 23: 1.

Moënne K, García C. Conflictos éticos en autoría de las publicaciones científicas del área médica. Rev Chil Radiol 2019; 25(3): 80-82.

*Correo electrónico: Karla Moënne / kmoenne@clinicalascondes.cl 